\title{
Natural noise and external wakefield seeding in a proton-driven plasma accelerator
}

\author{
K. V. Lotov, ${ }^{1,4}$ G. Z. Lotova, ${ }^{2,4}$ V. I. Lotov, ${ }^{3,4}$ A. Upadhyay, ${ }^{5}$ T. Tückmantel, ${ }^{5}$ A. Pukhov, ${ }^{5}$ and A. Caldwell ${ }^{6}$ \\ ${ }^{1}$ Budker Institute of Nuclear Physics SB RAS, 630090, Novosibirsk, Russia \\ ${ }^{2}$ Institute of Computational Mathematics and Mathematical Geophysics SB RAS, 630090, Novosibirsk, Russia \\ ${ }^{3}$ Sobolev Institute of Mathematics SB RAS, 630090, Novosibirsk, Russia \\ ${ }^{4}$ Novosibirsk State University, 630090, Novosibirsk, Russia \\ ${ }^{5}$ Institut für Theoretische Physik I, Heinrich-Heine-Universität Düsseldorf, 40225 Germany \\ ${ }^{6}$ Max-Planck-Institut für Physik, 80805, München, Germany
}

(Received 8 June 2012; published 2 April 2013)

\begin{abstract}
An accurate description of noise levels is of crucial importance for the correct simulation of instabilitydriven processes, such as the density modulation of a long proton bunch traversing a plasma. To insure that the correct instability develops, a seed field must be larger than the cumulative shot noise. We develop an analytical theory of the noise field and compare it with multidimensional simulations. We find that the natural noise wakefield generated in a plasma by the CERN Super Proton Synchrotron bunches is very low, at the level of $10 \mathrm{kV} / \mathrm{m}$. This fortunate fact eases the requirements on the seed. Our threedimensional simulations show that even a few tens $\mathrm{MeV}$ electron bunch precursor of a very moderate intensity is sufficient to seed the proton bunch self-modulation in plasma.
\end{abstract}

PACS numbers: 41.75.Lx, 52.35.Qz, 52.40.Mj

Plasma wakefield acceleration offers tremendous potential for much more compact, and therefore much more cost effective, particle accelerators. Many variants of beam-driven plasma wakefield acceleration have been considered, as discussed below. Sophisticated simulation programs are the key to understanding the physics of plasma wakefield accelerators and serve to guide design choices. To be reliable, these simulations must reproduce the basic underlying physical situation. Since it is not possible today to individually simulate the large number of particles in a typical bunch of particles from accelerators, approximations must be made, such as using "macroparticles" to represent the effect of a large number of individual particles. Effects that depend on the random placement of the particles in a bunch are then greatly exaggerated in the simulations, and can lead to faulty conclusions. In this paper, we evaluate the random, or "shot," electric field distribution created by the passage of a long proton bunch in a plasma, and we explain how the correct level of noise (which is much smaller than that normally simulated) can be introduced into simulation codes.

Particle beam-driven plasma wakefield acceleration (PWFA) is capable of producing accelerating gradients far in excess of those in conventional accelerators [1], but it needs the drive beam to be properly shaped or compressed (see, e.g., Ref. [2] for PWFA basics). So far, PWFA has been experimentally studied with electron or positron

Published by the American Physical Society under the terms of the Creative Commons Attribution 3.0 License. Further distribution of this work must maintain attribution to the author( $s)$ and the published article's title, journal citation, and DOI. beams shaped before the plasma [1,3-10]. Recently a new approach was proposed [11-13] that assumes beam shaping by the plasma itself as a result of the transverse twostream beam-plasma instability [14-17]. At the nonlinear stage, the instability splits the initially long beam into short bunches spaced exactly one plasma wavelength apart $[18,19]$. Harnessing the instability would make it possible to excite strong wakefields by initially long beams without a complicated and expensive compressor or chopper. The controlled instability is the key physical effect to be demonstrated by the proposed proton-driven PWFA experiment at CERN [19,20] and auxiliary experiments [21,22].

To be useful for acceleration of a witness beam, the generated wake must be axisymmetric. Yet, the plasma supports various modes of the instability, including nonaxisymmetric (or hosing) modes. The latter are undesirable since they quickly destroy the beam to the state at which no strong wakefield is excited [12]. Fortunately, if the axisymmetric mode has grown up to sufficiently high amplitude, it prevents development of other modes [11]. Simulations show that the proper mode may dominate when an externally introduced seed perturbation is used [12]. The amplitude of the perturbation must be much higher than the noise level from which hosing modes grow up. To simulate the instability correctly and to determine the required amplitude of the seed perturbation, we have to know the noise level.

There could be various sources of uncontrollable seed perturbations for instabilities. The one we are interested in is the shot noise of individual beam particles. The wakefield pattern in this case moves with the beam and will be amplified by the beam instability.

The shot noise field is a sum of wakefields left behind by separate beam particles. The contribution of a single proton 
located at the radius $r_{b}$ to the on-axis wakefield at the distance $z_{b}$ downstream can be taken from Ref. [23]:

$$
\begin{aligned}
E_{b z} & =-2 e k_{p}^{2} K_{0}\left(k_{p} r_{b}\right) \cos \left(k_{p} z_{b}\right), \\
\left|E_{b \perp}\right| & =2 e k_{p}^{2} K_{1}\left(k_{p} r_{b}\right)\left|\sin \left(k_{p} z_{b}\right)\right|,
\end{aligned}
$$

where $k_{p}=\omega_{p} / c$ is the plasma wave number determined by the plasma frequency $\omega_{p}$ and the light velocity $c, e>0$ is the elementary charge, and $K_{0}$ and $K_{1}$ are the modified Bessel functions of the second kind. We use cylindrical coordinates with the $z$ axis being the direction of beam propagation.

We will illustrate the obtained formulas using the beam and plasma parameters discussed in Ref. [19] as a SPSLHC variant: the number of protons in one bunch $N=$ $1.15 \times 10^{11}$, bunch radius $\sigma_{r}=k_{p}^{-1}=0.2 \mathrm{~mm}$, and bunch length $\sigma_{z}=12 \mathrm{~cm}$. The plasma density is taken to be $n_{p}=7 \times 10^{14} \mathrm{~cm}^{-3}$ to satisfy $k_{p} \sigma_{r}=1$.

First we calculate the longitudinal noise field $E_{n z}$ on axis. As the beam evolves slowly compared to the plasma time scale, the field depends on longitudinal coordinate and time in the combination $\xi=z-c t$. We consider the plasma wakefields at different positions $z_{0}$ for a fixed time. The field can be presented as the real part of some complex function:

$$
E_{n z}\left(z_{0}\right)=\operatorname{Re}\left(E_{c z}\left(z_{0}\right) e^{i k_{p} z_{0}}\right) .
$$

Each beam particle located at $z>z_{0}$ makes a contribution $Q_{j}$ into the complex amplitude $E_{c z}$, which depends on particle location with respect to the observation point. The absolute value of the contribution depends on $r_{b}$, while the argument is determined by $z_{b}=z-z_{0}$. The process of noise field excitation thus can be considered as a twodimensional random walk $\left\{R_{N}\right\}$ on the complex amplitude plane [Fig. 1(a)]: $R_{N}=Q_{1}+\ldots+Q_{N}$.

We imagine the protons to be labeled randomly, and in this case we have the sum of a large number of values of $Q_{j}$, each with the same probability distribution. The total field $E_{n z}$ can therefore be viewed as the result of a onedimensional random walk along the real axis with the step $E_{b z}$ given by Eq. (1). We will characterize this walk first.

The distribution of $E_{b z}$ has a finite variance, and we can therefore use the central limit theorem to give us the


FIG. 1. (a) Excitation of the noise field as a random walk; (b) second moments of probability distributions. distribution of $E_{n z}$. It is a Gaussian distribution centered at $N\left\langle E_{b z}\right\rangle$ with a variance $\sigma_{n z}^{2}=N \sigma_{b z}^{2}$, where $N$ is the number of protons contributing to the field and $\sigma_{b z}^{2}$ is the variance of the distribution of $E_{b z}$. The average field $\left\langle E_{b z}\right\rangle$ is exponentially suppressed for a smooth Gaussian beam, and is for our purposes zero. We therefore focus on the width of the distribution, given by $\sigma_{b z}^{2}$. We evaluate this as follows:

$$
\sigma_{b z}^{2} \approx 4 e^{2} k_{p}^{4}\left\langle K_{0}\left(k_{p} r\right)^{2}\right\rangle_{p(r)}\left\langle\cos \left(k_{p} z_{b}\right)^{2}\right\rangle_{p(z)}
$$

where $\langle\ldots\rangle_{p(\circ)}$ means averaging according to probability distribution function $p(\circ)$. The probability density distribution for single protons is described by the Gaussian functions

$$
\begin{aligned}
& p(r) d r=\frac{r}{\sigma_{r}^{2}} e^{-r^{2} / 2 \sigma_{r}^{2}} d r, \\
& p(z) d z=\frac{1}{\sqrt{2 \pi} \sigma_{z}} e^{-z^{2} / 2 \sigma_{z}^{2}} d z .
\end{aligned}
$$

We have

$$
\left\langle\cos \left(k_{p} z_{b}\right)^{2}\right\rangle_{p(z)}=\int_{z_{0}}^{\infty} \frac{\cos ^{2}\left(k_{p}\left(z-z_{0}\right)\right)}{\sqrt{2 \pi} \sigma_{z}} e^{-z^{2} / 2 \sigma_{z}^{2}} d z \approx \frac{1}{2},
$$

and

$$
\begin{aligned}
\left\langle K_{0}\left(k_{p} r\right)^{2}\right\rangle_{p(r)} & =\frac{1}{k_{p}^{2} \sigma_{r}^{2}} \int_{0}^{\infty} x K_{0}^{2}(x) e^{-x^{2} / 2 k_{p}^{2} \sigma_{r}^{2}} d x \\
& \equiv \frac{\tilde{b}_{0}\left(k_{p} \sigma_{r}\right)}{k_{p}^{2} \sigma_{r}^{2}}
\end{aligned}
$$

so that

$$
\sigma_{b z}^{2} \approx 2 e^{2} k_{p}^{2} \tilde{b}_{0} / \sigma_{r}^{2}
$$

The function $\tilde{b}_{0}\left(k_{p} \sigma_{r}\right)$ is shown in Fig. 1(b). For our example parameter set [24], $\tilde{b}_{0} \approx 0.35$, which gives the root mean square (rms) of the shot noise field

$$
E_{\mathrm{z}, \mathrm{rms}}=\sqrt{N} \sigma_{b z} \approx 10 \mathrm{kV} / \mathrm{m} .
$$

The rms of the field at a certain position is difficult to extract from simulations. The oscillating field excited by the beam is usually characterized by the amplitude, that is by $\left|E_{c z}\left(z_{0}\right)\right|$. To find the expectation $E_{a z}$ for $\left|E_{c z}\left(z_{0}\right)\right|$, we return to the two-dimensional random walk shown in Fig. 1(a). It is known that if the distribution of vector projections weakly converges to the one-dimensional standard normal distribution for each chosen direction, then the distribution of the vector weakly converges to the twodimensional standard normal distribution (the theorem of Cramér-Wold [25]). Thus 


$$
\begin{aligned}
E_{a z} & =\sqrt{N} \sigma_{b z} \frac{1}{2 \pi} \int_{0}^{\infty} r e^{-r^{2} / 2} 2 \pi r d r=\sqrt{\frac{\pi}{2}} E_{\mathrm{z}, \mathrm{rms}} \\
& =\frac{e k_{p}}{\sigma_{r}} \sqrt{\pi N \tilde{b}_{0}\left(k_{p} \sigma_{r}\right)} .
\end{aligned}
$$

In our case, $E_{a z} \approx 13 \mathrm{kV} / \mathrm{m}$.

Figure 2 illustrates example noise fields along the proton bunch axis as well as the expectation from our calculations. The examples are from LCODE [26] simulations of the plasma response to the full Super Proton Synchrotron (SPS) beam [19]. The five thin curves correspond to five different initializations of the random number generator. The thick curve is the expectation from our calculations. The dotted curve is the average over 60 different initializations of the random number generator.

The expectation for the off-axis longitudinal electric field can be found in a similar way, but with a more complicated probability density distribution used instead of (3). Alternatively, the expectation can be obtained by a Monte Carlo simulation of a random walk (Fig. 3). Note that the field area is wider than the beam itself.

Since the electric field excited in the plasma is described by a potential function, we can find the transverse field component from the longitudinal one through the potential $\Phi$ :

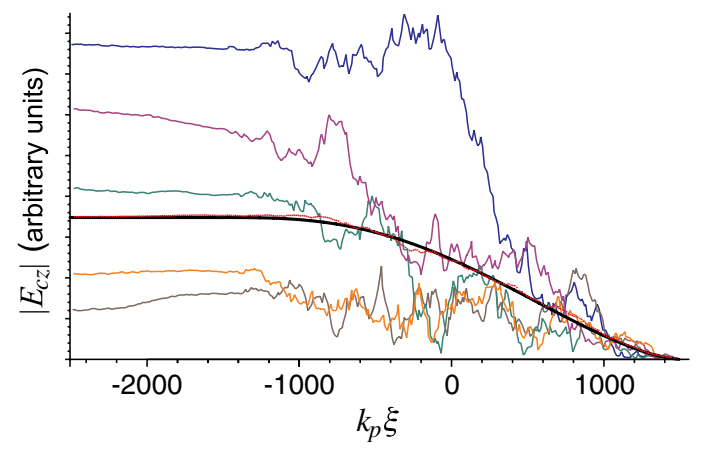

FIG. 2. Simulated field amplitudes for various beam ensembles (thin colored lines), the expectation value (black thick smooth line), and the field amplitude averaged over 60 beam ensembles (red dotted line) for LCODE simulations of a SPS beam.

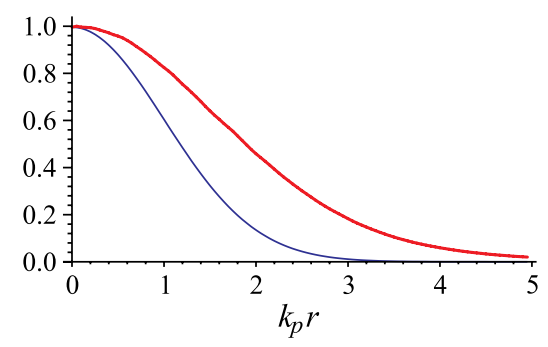

FIG. 3. The ratio of the expectation value $E_{a z}(r)$ to the value on axis, $E_{a z}(0)$, for $\sigma_{r}=k_{p}^{-1}$ (thick line) and the corresponding radial profile of the beam density (thin line).

$$
E_{z}=-\frac{\partial \Phi}{\partial z}=-i k_{p} \Phi, \quad \vec{E}_{\perp}=-\frac{\partial \Phi}{\partial \vec{r}_{\perp}}=\frac{1}{i k_{p}} \frac{\partial E_{z}}{\partial \vec{r}_{\perp}}
$$

Typical portraits of the potential $\Phi$ in the plane $(x, \xi)$ are shown in Fig. 4. These graphs are obtained from the random walk model by summing up contributions of separate test macroparticles for three different beam ensembles. We see that the transverse scale of the potential change is shorter than the longitudinal scale. Consequently, typical transverse fields must be higher than the longitudinal ones.

The total transverse field for a point on axis is

$$
E_{n \perp}=\sum_{b}\left|E_{b \perp}\right| \cos \phi_{b},
$$

where $\phi_{b}$ is an azimuthal angle of the vector connecting the sampling point and the proton $b$. To find the rms transverse noise field, we need to evaluate

$$
\sigma_{b \perp}^{2}=4 e^{2} k_{p}^{4}\left\langle K_{1}\left(k_{p} r\right)^{2}\right\rangle_{p(r)}\left\langle\sin \left(k_{p} z_{b}\right)^{2}\right\rangle_{p(z)}\left\langle\cos \left(\phi_{b}\right)^{2}\right\rangle_{p(\phi)} \text {. }
$$

The last two expectation values are easily calculated, but the expectation of the square of the Bessel function $\left\langle K_{1}\left(k_{p} r\right)^{2}\right\rangle_{p(r)}$ diverges. Here we need to limit the integration interval by some minimum radius $r_{\text {min }}$ to avoid divergence. At smaller radii, formula (2) is no longer valid. Several factors could limit the applicability of the linear cold fluid model used in derivation of (2).

A nonzero temperature $T_{e} \sim 5 \mathrm{eV}$ of plasma electrons modifies the plasma response on the scale of the Debye length

$$
r_{d} \approx 743 \sqrt{T_{e}(\mathrm{eV}) / n_{p}\left(\mathrm{~cm}^{-3}\right)} \sim 6 \times 10^{-5} \mathrm{~cm} .
$$

Plasma continuity and linearity of plasma response can be shown to break at much shorter scales [27].

The nonzero temperature also results in the nonzero group velocity $v_{g}=3 \omega_{p} r_{d}^{2} k$ of plasma waves that depends on the wave number $k$ of the perturbation. The sharply localized field spike (2) behind the proton is produced by short-wavelength wave harmonics, with transverse wave numbers $k \sim r_{v}^{-1}$ being responsible for the field spike in the area of the transverse size $r_{v}$. The higher $k$ the faster the energy drifts out of the field spike. Assuming the time of field evolution $\sigma_{z} / c$, we find the minimum scale $r_{v}$ for which the wave has no time to drift out of the field spike:

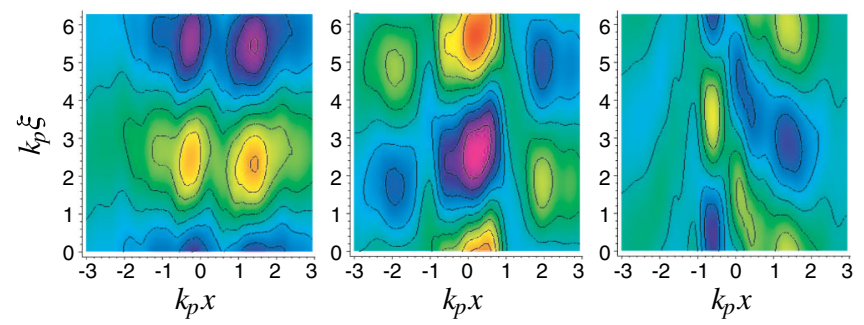

FIG. 4. Maps of the wakefield potential for three different beam ensembles. 


$$
\begin{aligned}
v_{g} \sigma_{z} / c & =r_{v}, \\
r_{v} & =\sqrt{3 k_{p} r_{d}^{2} \sigma_{z}} \approx 3 \times 10^{-3} \mathrm{~cm} \approx 0.1 k_{p}^{-1} .
\end{aligned}
$$

This is the scale we take for $r_{\min }$. Using this lower cutoff, we introduce

$$
\begin{aligned}
\left\langle K_{1}\left(k_{p} r\right)^{2}\right\rangle_{p(r)} & =\frac{1}{k_{p}^{2} \sigma_{r}^{2}} \int_{r_{\min }}^{\infty} x K_{1}^{2}(x) e^{-x^{2} /\left(2 k_{p}^{2} \sigma_{r}^{2}\right)} d x \\
& \equiv \frac{2 \tilde{b}_{1}\left(k_{p} \sigma_{r}\right)}{k_{p}^{2} \sigma_{r}^{2}}
\end{aligned}
$$

[Fig. 1(b)] and find

$$
E_{a \perp}=\frac{e k_{p}}{\sigma_{r}} \sqrt{\pi N \tilde{b}_{1}\left(k_{p} \sigma_{r}\right)} .
$$

For our parameter set, $E_{a \perp} \approx 20 \mathrm{kV} / \mathrm{m}$. An uncertainty in the determination of $r_{\min }$ has a little effect on the field expectation because of the weak (logarithmic) dependence of $\tilde{b}_{1}$ on $r_{\min }$. A factor of 2 smaller $r_{\min }$ results in a $0.5 \ln 2$ (or $40 \%$ ) increase in $\tilde{b}_{1}$ and a $20 \%$ increase of $E_{a \perp}$.

To simulate the shot noise properly, the following method of initial particle positioning was developed and introduced into the hybrid 3D particle-in-cell code VLPL $[28,29]$. Since the number of numerical macroparticles is several orders of magnitude smaller than the $1.15 \times$ $10^{11}$ protons in the SPS bunch, the noise generated by randomly seeded macroparticles would be significantly higher than the natural expectation (8). For this reason, we initialize all the numerical beam macroparticles at the center of the grid cells and distribute their transverse momenta regularly and symmetrically according to the bunch divergence. The longitudinal momenta have a random Gaussian distribution centered at $450 \mathrm{GeV} / c$ and the spread corresponding to the given longitudinal emittance. A bunch initialized in this way generates an extremely low numerical noise field (Fig. 5), 3 orders of magnitude below the expectation (8). This figure also gives an idea of how small is the contribution of numerical errors present in the PIC code to the noise level.

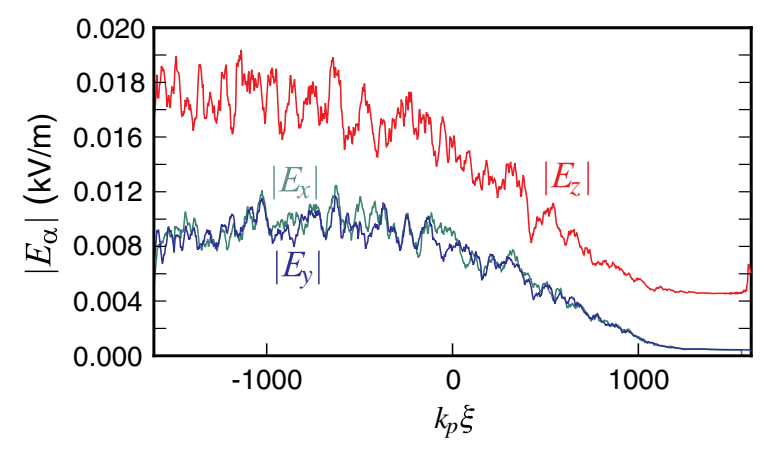

FIG. 5. Components of the on-axis electric field for the regular distribution of beam macroparticles.
To adjust the noise to the natural level (8), we introduce a random displacement $\epsilon$ to the regular positions of macroparticles in the configuration space:

$$
\epsilon=\delta r / h=\sqrt{N_{P} / N_{B}} \propto \sqrt{N_{P}} \delta n_{B} / n_{B} .
$$

Here $r$ is the space coordinate, $h$ is the cell size, $N_{P}$ is the number of numerical macroparticles that substitute for $N_{B}$ real beam protons in the cell volume, and $n_{B}$ is the local beam density. The random displacements (16) lead to the same level of numerical beam density fluctuations as the $\sqrt{N_{B}}$ fluctuations of the real beam proton number within the cell volume. Figure 6 illustrates that this random displacement indeed generates noise fields with the amplitude close to the calculated values.

Figure 7 shows simulated 3D evolutions of the SPS bunch for both the "unseeded" case, when the wakefield grows from the shot noise, and the case when the proton bunch self-modulation is seeded by a precursor electron bunch. The frame Fig. 7(a) gives the wakefield growth with propagation distance. The broken line corresponds to the simulation without any external seeding. The proton bunch self-modulation started to grow from the natural beam density fluctuations due to the shot noise. We see that it takes $10 \mathrm{~m}$ of plasma for the wake to reach its maximum at about $80 \mathrm{MV} / \mathrm{m}$. At the distance of $10 \mathrm{~m}$, the proton bunch
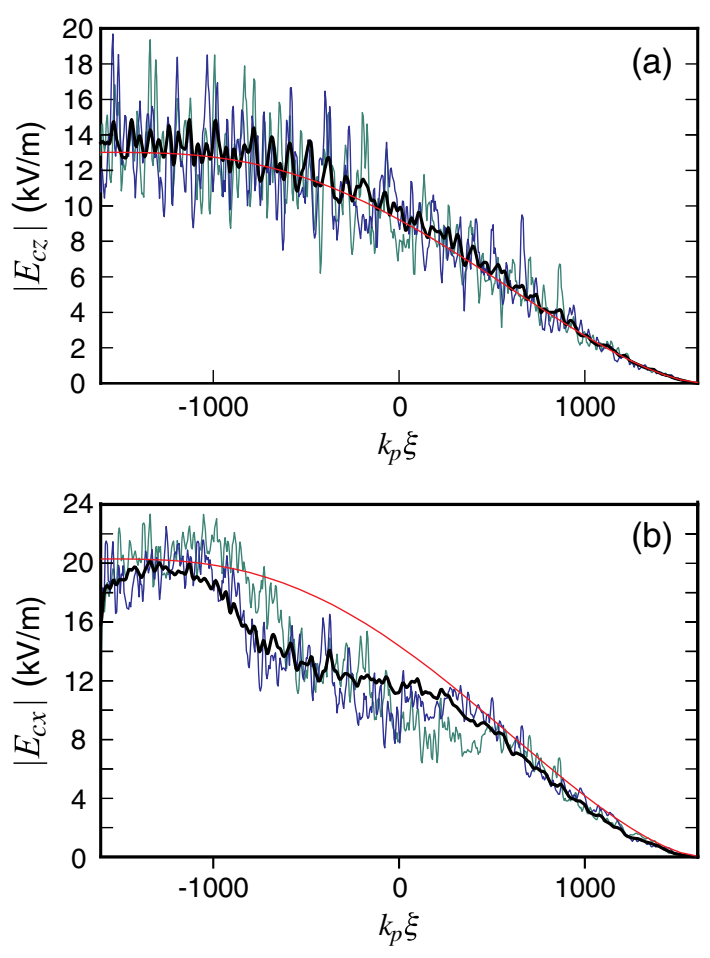

FIG. 6. Amplitudes of (a) longitudinal $E_{z}$ and (b) transverse $E_{x}$ electric field on the axis for the controlled random displacement of beam macroparticles. Thin colored lines are simulated fields for various beam ensembles, black thick lines are averages over 5 beam ensembles, and red lines are calculated expectation values. 


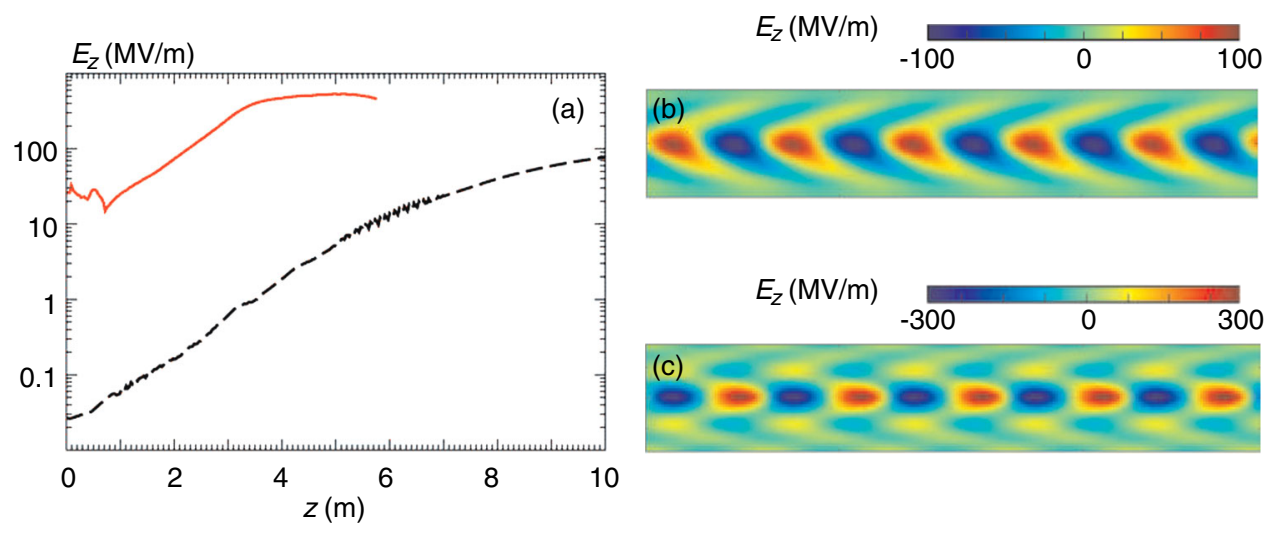

FIG. 7. (a) Growth of the accelerating field with propagation distance in plasmas. The broken line corresponds to an unseeded SPS proton bunch; the solid line shows the self-modulation growth when a $10 \mathrm{MeV}$ electron beam has been used as a precursor. (b) $2 \mathrm{D}$ onaxis cut of the wakefield in the unseeded simulation behind the driver at the distance $z=9 \mathrm{~m}$. The field is asymmetric due to the competition between hosing and self-modulation. (c) $2 \mathrm{D}$ on-axis cut of the wakefield in the seeded simulation behind the driver at the distance $z=4 \mathrm{~m}$. The wake is symmetric.

has already significantly diffracted due to its transverse emittance. The generated wakefield behind the proton bunch is shown in Fig. 7(b). We zoom here at the position $2 \sigma_{z}$ behind the middle of the SPS bunch. One sees that the wake is not very symmetric and is tilted. This tilt is due to the presence of the hosing instability that competes with the axisymmetric self-modulational mode [12]. The solid red line in Fig. 7(a) shows the wakefield growth when the self-modulation is seeded by a precursor electron bunch. We used a $10 \mathrm{MeV}$ electron bunch of 1 ps duration and the current of $100 \mathrm{~A}$ as the precursor. It is seen that the field fluctuates strongly at the first meter of propagation in plasma. This is due to strong self-focusing and defocusing of the electron bunch. After the first meter, the selfmodulation of the proton bunch sets in and the field starts to grow exponentially. The maximum field of some $0.6 \mathrm{GV} / \mathrm{m}$ is reached after $4 \mathrm{~m}$ propagation distance. The field snapshot at this distance is given in Fig. 7(c). Apparently, the field is symmetric so that the selfmodulation mode wins over the hosing. Our simulations also show that the growth of self-modulation is not very sensitive to the electron bunch parameters such as the energy and the current. It is important that the initial wake generated by the precursor is significantly larger than the noise field of the proton bunch and that the saturation of the self-modulation is reached before the proton bunch diffracts away due to its transverse emittance.

We have derived expressions for the noise fields present in long bunches traversing a plasma. Evaluating these for the parameters of the proton bunches at the CERN SPS accelerator, we find that the fields are at the $10 \mathrm{kV} / \mathrm{m}$ scale. This scale is far below the noise fields typically realized in particle-in-cell codes used to simulate the process. We have described a procedure for introducing the correct noise levels in the simulations, and we have shown that even a $10 \mathrm{MeV}$ electron bunch of 1 ps duration and maximum current of $100 \mathrm{~A}$ is sufficient to seed the axisymmetric mode of the self-modulation.

\section{ACKNOWLEDGMENTS}

This work is supported by the Ministry of Education and Science of Russia (Projects No. 14.B37.21.0784, No. 14.B37.21.0750, and No. 8387) and by RFBR Grants (No. 11-01-00249, No. 11-02-00563, and No. 12-0100727).

[1] I. Blumenfeld et al., Nature (London) 445, 741 (2007).

[2] E. Esarey, P. Sprangle, J. Krall, and A. Ting, IEEE Trans. Plasma Sci. 24, 252 (1996).

[3] A. K. Berezin et al., JETP Lett. 13, 354 (1971); Plasma Phys. Rep. 20, 596 (1994).

[4] J. Rosenzweig, D. Cline, B. Cole, H. Figueroa, W. Gai, R. Konecny, J. Norem, P. Schoessow, and J. Simpson, Phys. Rev. Lett. 61, 98 (1988); J. Rosenzweig, P. Schoessow, B. Cole, W. Gai, R. Konecny, J. Norem, and J. Simpson, Phys. Rev. A 39, 1586 (1989); J. B. Rosenzweig, P. Schoessow, B. Cole, C. Ho, W. Gai, R. Konecny, S. Mtingwa, J. Norem, M. Rosing, and J. Simpson, Phys. Fluids B 2, 1376 (1990).

[5] K. Nakajima, A. Enomoto, H. Kobayashi, H. Nakanishi, Y. Nishida, A. Ogata, S. Ohsawa, T. Oogoe, T. Shoji, and T. Urano, Nucl. Instrum. Methods Phys. Res., Sect. A 292, 12 (1990); A. Ogata, AIP Conf. Proc., 279, 420 (1993).

[6] N. Barov, J. Rosenzweig, M. Conde, W. Gai, and J. Power, Phys. Rev. ST Accel. Beams 3, 011301 (2000); N. Barov et al. in, Particle Accelerator Conference, Chicago, IL, 2001 (IEEE, New York, 2001), pp. 126-128.

[7] S. J. Russell, J. D. Goettee, and B. E. Carlsten in, Particle Accelerator Conference, Chicago, IL, 2001 (IEEE, New York, 2001), pp. 3975-3977.

[8] V. Yakimenko et al., Phys. Rev. Lett. 91, 014802 (2003). 
[9] E. Kallos, T. Katsouleas, W. Kimura, K. Kusche, P. Muggli, I. Pavlishin, I. Pogorelsky, D. Stolyarov, and V. Yakimenko, Phys. Rev. Lett. 100, 074802 (2008); P. Muggli et al. in, Proceedings of the 2011 Particle Accelerator Conference, New York, NY, 2011 (IEEE, New York, 2011), TUOBN3.

[10] C. Joshi et al. Phys. Plasmas 9, 1845 (2002); B. E. Blue et al., Phys. Rev. Lett. 90, 214801 (2003); P. Muggli et al., Phys. Rev. Lett. 93, 014802 (2004); M. J. Hogan et al., Phys. Rev. Lett. 95, 054802 (2005); P. Muggli et al., Phys. Rev. Lett. 101, 055001 (2008); N. Kirby et al., Phys. Rev. ST Accel. Beams 12, 051302 (2009).

[11] K. V. Lotov, Proceedings of the 6th European Particle Accelerator Conference, Stockholm, 1998 (IOP, London, 1998), pp. 806-808.

[12] N. Kumar, A. Pukhov, and K. Lotov, Phys. Rev. Lett. 104, 255003 (2010).

[13] A. Caldwell, K. Lotov, A. Pukhov, and G. Xia, Plasma Phys. Controlled Fusion 53, 014003 (2011).

[14] J. Krall and G. Joyce, Phys. Plasmas 2, 1326 (1995).

[15] D. H. Whittum, Phys. Plasmas 4, 1154 (1997).

[16] K. V. Lotov, Nucl. Instrum. Methods Phys. Res., Sect. A 410, 461 (1998).
[17] A. Pukhov, N. Kumar, T. Tückmantel, A. Upadhyay, K. Lotov, P. Muggli, V. Khudik, C. Siemon, and G. Shvets, Phys. Rev. Lett. 107, 145003 (2011).

[18] K. V. Lotov, Phys. Plasmas 18, 024501 (2011).

[19] A. Caldwell and K. V. Lotov, Phys. Plasmas 18, 103101 (2011).

[20] G. Xia et al., Proceedings of the 2011 Particle Accelerator Conference, New York, NY, 2011 (IEEE, New York, 2011), TUOBN5.

[21] J. Vieira, Y. Fang, W. B. Mori, L. O. Silva, and P. Muggli, Phys. Plasmas 19, 063105 (2012).

[22] A. V. Petrenko, K. V. Lotov, P. V. Logatchov, and A. V. Burdakov, AIP Conf. Proc., 1229, 467 (2010).

[23] T. Katsouleas, S. Wilks, P. Chen, J. M. Dawson, and J. J. Su, Part. Accel. 22, 81 (1987).

[24] Note that the restriction on $r$ discussed in the next section is applied here also.

[25] P. Billingsley, Convergence of Probability Measures (Wiley and Sons, New York, 1968).

[26] K. V. Lotov, Phys. Plasmas 5, 785 (1998).

[27] K. V. Lotov et al., arXiv:1204.3444.

[28] A. Pukhov, J. Plasma Phys. 61, 425 (1999).

[29] T. Tuckmantel, A. Pukhov, J. Liljo, and M. Hochbruck, IEEE Trans. Plasma Sci. 38, 2383 (2010). 